\title{
Gridded Electron Guns and Modulation of Intense Beams
}

J. R. Harris, P. G. O'Shea

May 5, 2006

IEEE Transactions on Electron Devices 
This document was prepared as an account of work sponsored by an agency of the United States Government. Neither the United States Government nor the University of California nor any of their employees, makes any warranty, express or implied, or assumes any legal liability or responsibility for the accuracy, completeness, or usefulness of any information, apparatus, product, or process disclosed, or represents that its use would not infringe privately owned rights. Reference herein to any specific commercial product, process, or service by trade name, trademark, manufacturer, or otherwise, does not necessarily constitute or imply its endorsement, recommendation, or favoring by the United States Government or the University of California. The views and opinions of authors expressed herein do not necessarily state or reflect those of the United States Government or the University of California, and shall not be used for advertising or product endorsement purposes. 
Gridded Electron Guns and Modulation of Intense Beams

\author{
J.R. Harris, Member, IEEE \\ Lawrence Livermore National Laboratory \\ 7000 East Avenue, Mail Stop L-645, Livermore, CA 94551
}

Ph: 925-424-4434, Fax: 925-422-1767, email: harris89@1lnl.gov

P.G. O'Shea, Fellow, IEEE

Department of Electrical and Computer Engineering

University of Maryland, College Park, MD 20740

email: poshea@umd.edu 


\begin{abstract}
Gridded guns are useful for producing modulated electron beams. This modulation is generally limited to simple gating of the beam, but may be used to apply structure to the beam pulse shape. In intense beams, this structure spawns space charge waves whose dynamics depend in part on the relative strengths of the velocity and density variations which comprise the initial current modulation. In this paper, we calculate the strengths of beam current and velocity modulation produced in a gridded electron gun, and show that under normal conditions the initial modulation is dominated by density variation rather than velocity variation.
\end{abstract}

Index Terms

Particle beams, electron guns, space charge waves, electron tubes.

This work was supported in part by the Department of Energy, the Office of Naval Research, and the Directed Energy Professional Society.

This work was performed in part under the auspices of the U. S. Department of Energy by University of California, Lawrence Livermore National Laboratory under contract W-7405-Eng-48. 


\section{INTRODUCTION}

Advanced accelerator applications, such as intense x-ray sources, future linear colliders, and heavy ion fusion, increasingly require high current, low emittance beams. The beams used in many of these applications are ultimately accelerated to relativistic energy, but they are all nonrelativistic and dominated by space charge forces when initially created. These space charge forces can drive instabilities and inhomogeneities that can lead to emittance growth, coherent synchrotron radiation, and other disruptive phenomena, even after the beam becomes relativistic. Heavy ion fusion presents unique challenges, as it requires the propagation of intense [1] nonrelativistic beams over distances on the order of $1-10 \mathrm{~km}[2]$. These beams will be extremely susceptible to emittance growth and other difficulties associated with space charge waves launched from unintended modulation of the beam during generation and transport. These emerging applications are driving a renewed interest in intense beams and space charge waves.

In general, current perturbations which launch space charge waves may be due to variation in beam velocity, density, or both. The dynamics of the waves depend in part on the relative strength of the velocity and density contributions to the initial perturbation [3]. Work at the University of Maryland has focused on studying space charge wave propagation in the context of low energy electron beam transport systems. In this paper, we calculate the relative strengths of the velocity and current modulation that can be achieved in gridded Pierce-focusing [4] electron guns, and look at some consequences for beam dynamics. Nonrelativistic velocities, electron transit times which are fast compared 
to changes in applied voltages, and beam modulation wavelengths which are long compared to the beam pipe radius, will be assumed throughout.

\section{BEAM MODULATION}

\section{A. Gridded Guns.}

The techniques used for analysis of planar triodes are also useful for the analysis of nonconverging gridded guns $[5,6]$. Both gridded guns and triodes may be operated in three regimes: saturation, amplification, and cutoff. In saturation, the current is limited by space charge, and is governed by the Child-Langmuir equation [7]. If the magnitude of the grid voltage is increased sufficiently, the gun will be brought into triode amplification mode, where the current is governed by

$$
J=K_{T}\left(V_{g k}+\frac{V_{a k}}{\mu}\right)^{3 / 2},
$$

where $J$ is the current density produced from the cathode, $K_{T}$ is a constant depending on the geometry of the triode, $V_{g k}$ is the voltage between the cathode and grid, $V_{a k}$ is the voltage between the cathode and anode, and $\mu$ is the triode amplification factor [6]. If the magnitude of the grid voltage is increased even more so that

$$
V_{g k}+\frac{V_{a k}}{\mu} \leq 0,
$$

the gun will be driven into cutoff, so that no current will escape.

If the gun is operated in pulsed mode, the voltage applied between the grid and cathode will contain both a DC component and a pulsed component, as shown in Fig. 1. Normally, the strength of the pulser voltage and the DC bias voltage will be adjusted to drive the gun fully into cutoff or fully into saturation, producing a flat-top beam pulse. 
To deliberately produce a structured beam pulse, the bias voltage is adjusted to keep the gun in triode amplification mode at the top of the cathode pulse. The desired modulating signal is then applied between the grid and the cathode. This modulating signal may also arise accidentally, since any ringing, droop, or other structure on the pulse will be replicated in the current produced from the gun [5]. Such unintended structure is almost always present in the output signal of pulsed power systems, like the pulse forming lines frequently used to supply the pulsed voltage $V_{P}(t)$.

\section{B. Strength of Velocity Modulation}

Modulating the grid-cathode voltage as shown in Fig. 1 will result in a beam carrying velocity and density modulation, the strengths of which can be calculated [5]. For a nonrelativistic beam accelerated through a voltage $V_{0}$, the beam velocity is given by

$$
v_{0}=\sqrt{\frac{2 q V_{0}}{m}} .
$$

A perturbation $\Delta V(t)$ in the accelerating voltage will produce a perturbation of approximately

$$
v_{1}(t)=\sqrt{\frac{q}{2 m V_{0}}} \Delta V(t)
$$

in the beam velocity. The strength of this velocity perturbation can be written in dimensionless form as

$$
\delta=\frac{\left.v_{1}\right|_{\max }}{v_{0}},
$$


where $\left.v_{1}\right|_{\max }$ is the maximum value of the initial velocity perturbation. The velocity perturbation strength is therefore related to the applied voltage by

$$
\delta=\frac{\left.\Delta V\right|_{\max }}{2 V_{0}}
$$

The total (unperturbed) voltage through which the beam is accelerated in a gridded gun is the sum of the cathode-grid potential $V_{g k}$ and the grid-anode potential $V_{g a}$,

$$
V_{0}=V_{g k}+V_{g a}
$$

and (in the configuration of Fig. 1) the cathode-grid potential is the difference of a DC bias voltage $V_{B}$ and the cathode pulser voltage $V_{P}(t)$. Since the cathode pulser voltage is used to gate the beam, it will take two nominal values: zero when emission is suppressed, and a nonzero value $V_{P 0}$ when beam is being extracted. The accelerating voltage when beam is extracted is then

$$
V_{0}=V_{g a}+V_{P 0}-V_{B}
$$

If a perturbation is superimposed on $V_{P 0}$, then eq. (6) becomes

$$
\delta=\frac{\left.\Delta V_{P}\right|_{\max }}{2\left[V_{g a}+V_{P 0}-V_{B}\right]},
$$

where $\left.\Delta V_{P}\right|_{\max }$ is the maximum strength of the perturbation in the pulser voltage. C. Strength of Current Modulation.

The dimensionless current perturbation strength can be defined as the ratio of the maximum perturbation in beam current to the unperturbed current, measured as the beam exits the gun: 


$$
\eta=\frac{\left.I_{1}\right|_{\max }}{I_{0}}
$$

The current in a gridded gun operating in triode amplification mode is

$$
I=K_{T} A\left[V_{P}(t)-V_{B}+\frac{V_{g a}+V_{P}(t)-V_{B}}{\mu}\right]^{3 / 2},
$$

where $A$ is the cross-sectional area of the cathode. The acceleration voltage $V_{g a}$ is generally much greater than the control voltage $V_{P}(t)-V_{B}$, so that the control voltage may be neglected in the third term of eq. (11). Any perturbation in the pulser voltage will create a perturbation in the beam current, the maximum value of which is given by

$$
\left.\left.\Delta I\right|_{\max } \approx K_{T} A \frac{3}{2}\left(V_{P 0}-V_{B}+\frac{V_{g a}}{\mu}\right)^{1 / 2} \Delta V_{P}\right|_{\max } .
$$

The strength of the current perturbation in the beam exiting the gun is then given by

$$
\eta=\left.\frac{\left.\Delta I\right|_{\max }}{I} \approx \frac{3}{2}\left(V_{P 0}-V_{B}+\frac{V_{g a}}{\mu}\right)^{-1} \Delta V_{P}\right|_{\max } .
$$

D. Ratio of Modulation Strengths

The ratio of the current and velocity perturbation strengths is therefore given by

$$
\frac{\eta}{\delta}=3\left[\frac{V_{g a}+V_{P 0}-V_{B}}{\frac{V_{g a}}{\mu}+V_{P 0}-V_{B}}\right] .
$$

Since the control voltage is assumed to be much smaller than the accelerating voltage, this equation may be rewritten as 


$$
\frac{\eta}{\delta}=3\left[\frac{V_{g a}}{\frac{V_{g a}}{\mu}+V_{P 0}-V_{B}}\right] .
$$

Two special cases of this equation are of particular interest. First, when the grid and cathode are at the same potential, $V_{P 0}-V_{B}=0$, and the ratio of modulation strengths becomes equal to three times the amplification factor:

$$
\frac{\eta}{\delta}=3 \mu
$$

Second, as the gun approaches triode cutoff, the denominator in eq. (15) will approach zero, and $\eta / \delta \rightarrow \infty$. In both of these cases, the strength of the current perturbation exceeds that of the velocity perturbation. In fact, eq. (15) implies that the strength of the current modulation will always be much larger than the strength of the velocity modulation provided that

$$
\left(3-\frac{1}{\mu}\right) V_{g a} \gg V_{P 0}-V_{B} .
$$

This requirement holds in all conventional gridded guns.

Fig. 2 shows the ratio $\eta / \delta$, calculated from eq. (14), as a function of grid-cathode voltage normalized by grid-anode voltage. Curves are shown for several values of amplification factor. For a given amplification factor, making the control voltage more negative will reduce beam current and ultimately drive the gun into triode cutoff, such that $\eta / \delta \rightarrow \infty$. For an infinite amplification factor, cutoff occurs when the grid-cathode voltage is zero, while for an amplification factor of one, cutoff could only occur when the grid-cathode voltage is comparable to the accelerating voltage, violating our assumption 
that $V_{g a}>V_{P 0}-V_{B}$. However, within that assumption, $\eta / \delta \rightarrow 3$ for unity amplification factor. Note that for small grid-cathode voltages and amplification factors greater than about $5, \eta>>\delta$ and the beam modulation is dominated by density modulation.

\section{E. Space Charge Limited Operation}

The preceding discussion assumed that the gun was operating in triode amplification mode. If the gun is operating in space charge limited mode, modulating the grid-cathode voltage will still result in production of a modulated beam. Since electrons produced from the cathode must still travel through the grid and anode, the beam velocity will still be given by eq. (3) and eq. (8), and the velocity modulation will still be given by eq. (9). However, the current drawn from the gun in saturation is no longer given by eq. (11), but rather by the Child-Langmuir Law [6,7]

$$
I_{S}=K_{D} A V_{0}^{3 / 2}
$$

where $K_{D}$ is a constant depending on gun geometry, and the limiting voltage $V_{0}$ is the anode-cathode voltage of eq. (8). Small changes in the grid-cathode voltage will produce current perturbations with strength

$$
\eta_{S}=\left.\frac{\left.\Delta I\right|_{\max , S}}{I_{S}} \approx \frac{3}{2}\left(V_{P 0}-V_{B}+V_{g a}\right)^{-1} \Delta V_{P}\right|_{\max },
$$

and the ratio of current perturbation strength to velocity perturbation strength is

$$
\left.\frac{\eta}{\delta}\right|_{S}=3
$$

which is the same as for a gun of unity amplification factor operating in triode amplification mode. This is a result of the expressions for current perturbation strength in space charge limited mode and triode amplification mode, which differ only by the 
presence of a factor of $\mu$ in eq. (13). Since most gridded guns have relatively large amplification factors, the extraction of large current perturbations will be easier when operating in triode amplification mode rather than space charge limited mode.

F. Negative Transconductance

Based on the sign convention of Fig. 1, and within the limits of our assumptions, we have shown that the ratio $\eta / \delta$ generally varies between 3 and $+\infty$. This implies that $\eta / \delta$ is always positive. In order for $\eta / \delta$ to be negative, an increase in the pulser voltage -- or equivalently, a decrease in the bias voltage -- would decrease the current drawn from the gun. In other words, the gun's transconductance

$$
g_{m}=\frac{\partial I}{\partial V_{g k}}=\frac{\partial I}{\partial\left(V_{P}(t)-V_{B}\right)}
$$

would have to be negative. In theory, the transconductance of a triode is positive and varies as the one-third power of beam current [6]. However, a negative (or zero) transconductance can occur under certain conditions in practical electron guns. Fig. 3 shows current as a function of bias voltage measured on the University of Maryland Electron Ring gun [1], compared to a theoretical curve based on the ideal planar triode assumption [5]. For this test, the pulser voltage waveform remained unchanged but the bias voltage was varied. At $V_{B}=40 \mathrm{~V}$, the gun is in saturation. Increasing the bias voltage should bring the gun into triode amplification mode and reduce beam current. Instead, the current increases. This is believed to be caused by the use of an aperture plate to reduce the beam current, as shown in Fig. 1 [5]. As the bias voltage increases, current inside the gun will decrease according to eq. (11). The Pierce geometry is intended to balance the transverse space charge force in a full-current beam, but will 
cause the reduced-current beam to converge. The cross-sectional area of the beam decreases faster than its current, so that the current density will increase. As long as the beam radius is larger than the aperture radius, the current passing through the aperture will be the product of the aperture area and the incident beam density, so that the current passing through the aperture will initially increase as the bias voltage increases. Once the beam radius is smaller than the aperture radius, all of the current produced by the gun passes through the aperture, and continuing to increase the bias voltage causes the beam current to decrease. The result of this effect is that the gun has an operating region where it exhibits negative transconductance, allowing generation of waves with a negative (or zero) value of $\eta / \delta$. This may be useful for the production of some special space charge wave configurations discussed in Ref. [3].

\section{BEAM DYNAMICS}

\section{A. Space Charge Wave Velocity}

Modulation applied to an intense beam will evolve through the production of forward- and backward-traveling space charge waves, referred to as fast and slow waves [3]. This is true regardless of whether velocity modulation or density modulation dominates. It is also true regardless of the source of the modulation. Figs. 4 and 5 show examples of fast and slow wave propagation, and their effect on the beam current profile. In Fig. 4, the beam modulation is applied by modulating the grid voltage, while in Fig. 5 a laser pulse was applied to the cathode of the same gun to generate additional current by photoemission $[8,9]$. In both cases, the result is a density-dominated perturbation launching fast and slow space charge waves, and the differences seen in the evolving beams are due to differences in the shape of those initial perturbations [10]. 
Fast and slow waves travel in the beam frame at the velocity [11]

$$
c_{0}=\sqrt{\frac{q g I_{0}}{4 \pi \varepsilon_{0} m_{0} v_{0} \gamma^{5}}}
$$

where $q$ is the fundamental charge, $\varepsilon_{0}$ is the permittivity of free space, $m_{0}$ is the mass of the electron, $\gamma$ is the relativistic factor, and $I_{0}$ is the nominal beam current. The geometry factor $g=2 \ln (b / a)$ depends on the beam radius $(a)$ and the beam pipe radius ( $b$ ), and is generally of order one. The ratio of the wave speed to the beam velocity, in the nonrelativistic limit, is

$$
\frac{c_{0}}{v_{0}}=\sqrt{\frac{m_{0}^{1 / 2}}{2^{7 / 2} \pi \varepsilon_{0} q^{1 / 2}} g \frac{I_{0}}{V_{0}^{3 / 2}}} \approx 87 \sqrt{g \frac{I_{0}}{V_{0}^{3 / 2}}} .
$$

The quantity $I_{0} / V_{0}^{3 / 2}$ is the perveance of the gridded gun for a given value of the cathode-grid voltage. This equation indicates that the wave speed will be much smaller than the beam velocity except for unusually large values of $g$ and perveance.

\section{B. Density, Velocity, and Current Disturbances}

The fast and slow space charge waves consist of disturbances in beam density, velocity, and current, and the amplitudes of these disturbances depend on functions of $\eta$, $\delta, v_{0}$, and $c_{0}$. These disturbances are governed by the equations [3]

$$
\begin{aligned}
\lambda_{1}(z, t) & =-\frac{\lambda_{0}}{2}\left[\delta \frac{v_{0}}{c_{0}}-(\eta-\delta)\right] h\left(t-\frac{z}{v_{0}-c_{0}}\right) \\
& +\frac{\lambda_{0}}{2}\left[\delta \frac{v_{0}}{c_{0}}+(\eta-\delta)\right] h\left(t-\frac{z}{v_{0}+c_{0}}\right),
\end{aligned}
$$




$$
\begin{aligned}
v_{1}(z, t) & =\frac{v_{0}}{2}\left[\delta-(\eta-\delta) \frac{c_{0}}{v_{0}}\right] h\left(t-\frac{z}{v_{0}-c_{0}}\right) \\
& +\frac{v_{0}}{2}\left[\delta+(\eta-\delta) \frac{c_{0}}{v_{0}}\right] h\left(t-\frac{z}{v_{0}+c_{0}}\right),
\end{aligned}
$$

and

$$
\begin{aligned}
i_{1}(z, t)= & -\frac{i_{0}}{2}\left[\delta \frac{v_{0}}{c_{0}}-\eta+(\eta-\delta) \frac{c_{0}}{v_{0}}\right] h\left(t-\frac{z}{v_{0}-c_{0}}\right) \\
& +\frac{i_{0}}{2}\left[\delta \frac{v_{0}}{c_{0}}+\eta+(\eta-\delta) \frac{c_{0}}{v_{0}}\right] h\left(t-\frac{z}{v_{0}+c_{0}}\right),
\end{aligned}
$$

where the perturbed quantities are indicated by subscripts of one, the unperturbed quantities are indicated by subscripts of zero, and $h$ is a function varying between zero and one defining the shape of the modulation initially applied to the beam. The first half of each equation corresponds to the (backward-traveling) slow wave, and the second half of each equation corresponds to the (forward-traveling) fast wave. Note that in each case, the expressions for the amplitudes of the fast and slow waves differ slightly; this allows for the production of a fast wave only, or a slow wave only. Production of single space charge waves and other special cases of eqs. (24) - (26) are discussed in detail in Ref. [3].

In part II, we showed that when a conventional gridded electron gun is used to produce a modulated beam, the modulation is dominated by a density disturbance, rather than a velocity disturbance. Under what conditions is the initial velocity disturbance negligible for the purpose of the later space charge wave evolution?

This will be the case if, in the expressions governing the amplitudes of the fast and slow space charge waves, the magnitude of all the terms carrying a factor of $\delta$ is 
much less than the magnitude of all the terms carrying a factor of $\eta$. For example, for the initial velocity disturbance to be negligible for the evolution of the fast velocity wave,

$$
\left|\delta-\delta \frac{c_{0}}{v_{0}}\right|<<\left|\eta \frac{c_{0}}{v_{0}}\right|
$$

In the case of each of the fast space charge waves, this reduces to the requirement that

$$
\left|\frac{v_{0}}{c_{0}}-1\right|<<\frac{\eta}{\delta},
$$

while for each of the slow space charge waves, this reduces to the requirement

$$
\frac{v_{0}}{c_{0}}+1<<\frac{\eta}{\delta}
$$

If these requirements hold, the initial modulation can be treated as a pure density modulation, with no associated velocity disturbance. In this case, the expressions for the density, velocity, and current distributions simplify so that the fast and slow density waves have equal amplitudes and polarities, while the fast and slow velocity waves have equal amplitudes and opposite polarities. In addition, eq. (23) shows that $c_{0}<<v_{0}$ except in the case of unusually large values of $g$ and perveance; when this holds, the fast and slow current waves also have equal amplitude and polarity. These results are consistent with experimental observations $[12,13]$.

\section{DISCUSSION}

In this paper, we considered the production of modulated electron beams from gridded guns. It was assumed throughout that the beam was nonrelativistic, that the modulation was due to small changes in the cathode-grid voltage, that the beam dynamics were dominated by space charge forces rather than electron thermal velocities, that the beam produced in the gun then entered a transport system without subsequent 
acceleration, and that the beam modulation wavelengths were long compared to the beam pipe radius.

Under these assumptions, we showed:

1) that beam current modulation was predominately due to density modulation, rather than to velocity modulation;

2) that, except for guns with unusually high perveances and transport systems with unusually tightly confined beams, the velocity of space charge waves is much less than the beam velocity;

3) that if the requirements of eqs. (28) and (29) are fulfilled and $c_{0}<<v_{0}$, the velocity modulation initially imposed on the beam by the electron gun is totally negligible for the purpose of the later density, current, and velocity wave evolution in the beam;

4) that if all these conditions hold, the fast and slow density and current waves will have equal magnitude and polarity, while the fast and slow velocity waves will have equal magnitude and opposite polarity; and

5) that if the beam current is reduced by use of an aperture plate, then a gridded electron gun may exhibit negative transconductance behavior, allowing generation of waves with a negative (or zero) value of $\eta / \delta$. 


\section{REFERENCES}

[1] P. G. O'Shea, M. Reiser, R. A. Kishek, S. Bernal, H. Li, M. Preussner, V. Yun, Y. Cui, W. Zhang, Y. Zou, T. Godlove, D. Kehne, P. Haldemann, and I. Haber, "The University of Maryland Electron Ring (UMER)," Nucl. Instrum. Meth. A 464 646$652(2001)$.

[2] J. W. Kwan, "Experiments in Heavy Ion Fusion Beam Physics at LBNL, LLNL, and the University of Maryland," in Proceedings of the 1997 Particle Accelerator Conference.

[3] J. G. Wang, D. X. Wang, and M. Reiser, "Generation of space-charge waves due to localized perturbations in a space-charge dominated beam," Phys. Rev. Lett. 71, 1836$1839(1993)$.

[4] J. R. Pierce, "Rectilinear Electron Flow in Beams," J. Appl. Phys. 11, 548 (1940).

[5] J. R. Harris, Doctoral Dissertation, University of Maryland (2005). (Online: http://hdl.handle.net/1903/2906)

[6] K. R. Spangenberg, Vacuum Tubes. New York: McGraw-Hill, 1948.

[7] C. D. Child, "Discharge from Hot CaO," Phys. Rev. 4, 492-511 (1911).

[8] J.G. Neumann, J.R. Harris, B. Quinn, and P.G. O'Shea, "Production of photoemission-modulated beams in a thermionic electron gun," Rev. Sci. Instrum. 76, 033303 (2005).

[9] J. R. Harris, J. G. Neumann, and P. G. O'Shea, "Governing factors for production of photoemission-modulated electron beams," J. App. Phys. 99, 093306 (2006)

[10] J. R. Harris, J. G. Neumann, K. Tian, and P. G. O'Shea, to be published. 
[11] M. Reiser, Theory and Design of Charged Particle Beams, Wiley: New York (1994).

[12] J.R. Harris, J.G. Neumann, and P.G. O'Shea, "Modulation of Intense Beams in the University of Maryland Electron Ring," in Proc. 2005 Free-Electron Laser Conf., Stanford, CA, August 21-26, 2005.

[13] K. Tian, Y. Zou, Y. Cui, I. Haber, R. A. Kishek, M. Reiser, and P. G. O'Shea, "Experimental observations of longitudinal space-charge waves in intense electron beams," Phys. Rev. ST Accel. Beams 9, 014201 (2006). 
John R. Harris was born in Greenville, North Carolina. He received the B.S. degree in physics from Duke University in 2000, and the M.S. and Ph.D. degrees in electrical engineering from the University of Maryland in 2002 and 2005. He is currently a member of the Defense Sciences Engineering Division at Lawrence Livermore National Laboratory.

Patrick G. O'Shea (F'04) was born in Cork, Ireland. He received the B. Sc. degree in 1979 from University College Cork and the M.S. and Ph.D. degrees in 1982 and 1985, respectively, from the University of Maryland, College Park.

His early research was at the University of California, Los Alamos National Laboratory, on the Beam Experiment Aboard Rocket Project (BEAR), and the APEX Free-Electron Laser Project, and later at the Duke University Free-Electron Laser Laboratory. He rejoined the University of Maryland in 1999, and from 2001 to 2005 he was Director of the Institute for Research in Electronics and Applied Physics (IREAP). He is currently Chair of the Department of Electrical and Computer Engineering.

Prof. O'Shea is also a Fellow of the American Physical Society. 


\section{FIGURE CAPTIONS}

Fig. 1. Schematic of a high-perveance gridded electron gun [5]. Emission of electrons from the cathode $(\mathrm{K})$ is controlled by the potential on the grid $(\mathrm{G})$. Potential between the cathode and grid is maintained by a DC bias voltage $\left(\mathrm{V}_{\mathrm{B}}\right)$ and a pulsed voltage source $\left(\mathrm{V}_{\mathrm{P}}(\mathrm{t})\right)$, which is assumed to consist of oscillations superimposed on a rectangular pulse of height $\mathrm{V}_{\mathrm{P} 0}$. The grid and cathode are both held at negative potential with respect to the laboratory ground by the high voltage supply $\left(\mathrm{V}_{\mathrm{ga}}\right)$. Electrons escaping through the grid are accelerated toward the anode (A). Transverse expansion of the beam is prevented by Pierce electrodes $(\mathrm{P})$. Here, the anode is shown with a mesh to counteract aperture defocusing of the beam. An aperture plate (AP) is also shown, which can be included to adjust beam current external to the gun for beam transport experiments.

Fig. 2. Ratio of current modulation strength $\eta$ to velocity modulation strength $\delta$, from eq. (14). Independent variable is the grid-cathode (control) voltage as a fraction of the grid-anode (accelerating) voltage.

Fig. 3. Beam current as a function of bias voltage in the University of Maryland Electron Ring gridded gun [5]. Squares denote measured values, while the lower curve gives the expected current based on the ideal planar triode assumption and the amplification factor measured at cutoff. 
Fig. 4. Example of modulated beam in the University of Maryland Electron Ring [5, 12]. Modulation consists of sinusoidal fast and slow space charge waves, which interfere with each other as they move along the beam. Traces indicate current as a function of time, measured with beam position monitors at the indicated distances from the cathode.

Fig. 5. Discrete space charge wave propagation in the University of Maryland Electron Ring [12]. Beam energy was $10 \mathrm{keV}$ and flat top current was $38 \mathrm{~mA}$. Initial perturbation (P) generates fast (F) and slow (S) space charge waves of the same magnitude and polarity, which separate as the beam travels through the transport system. Measured wave separation indicates a wave velocity of $(1.6 \pm 0.14) \mathrm{Mm} / \mathrm{s}$. The perturbation shown here was generated by photomodulation [8]; however, like grid modulation from a conventional triode gun, this technique generates a density-dominated perturbation with negligible velocity modulation.

Reprinted from J.R. Harris, J.G. Neumann, and P.G. O'Shea, "Modulation of Intense Beams in the University of Maryland Electron Ring," in Proceedings of the 2005 FreeElectron Laser Conference. 
FIGURES

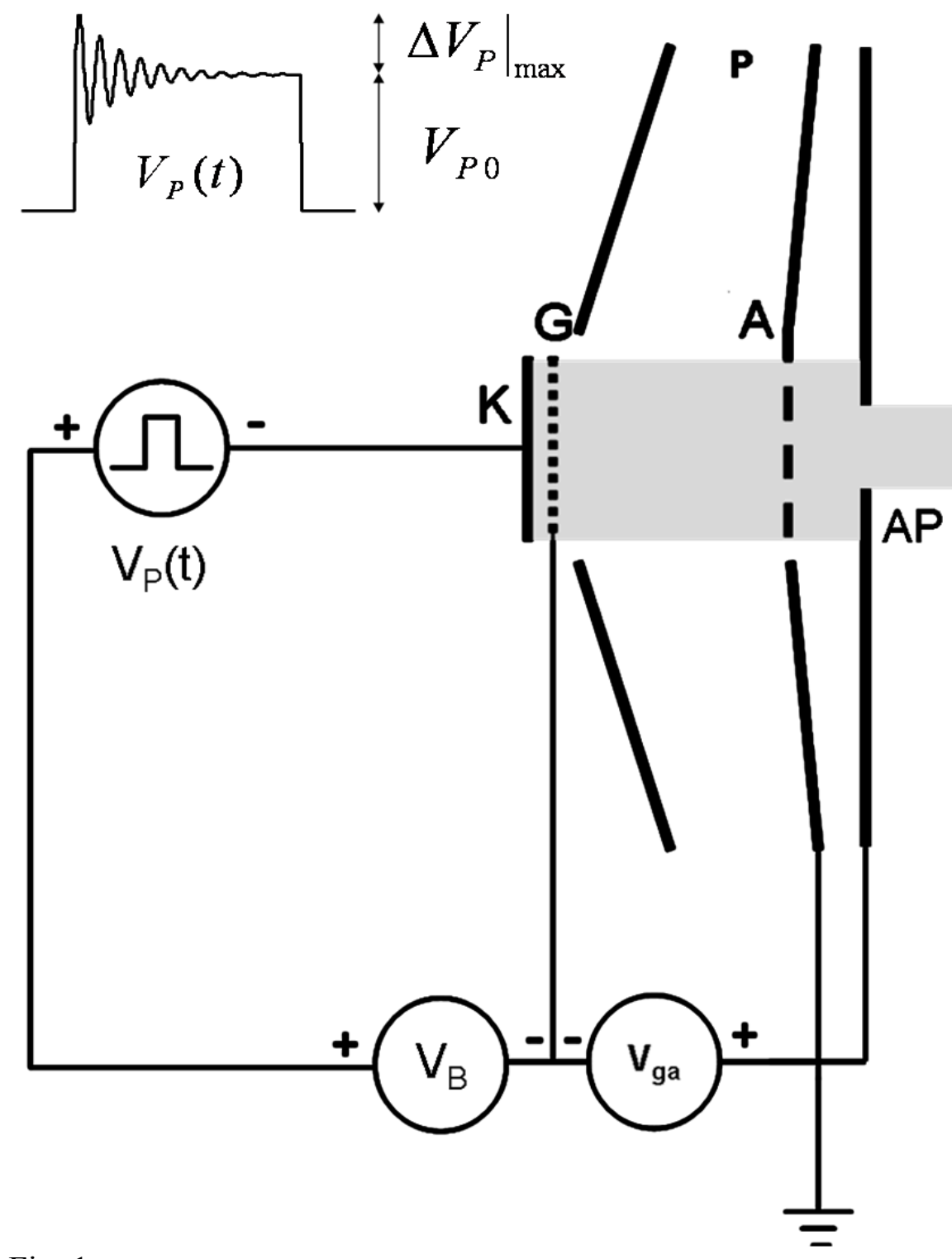

Fig. 1 


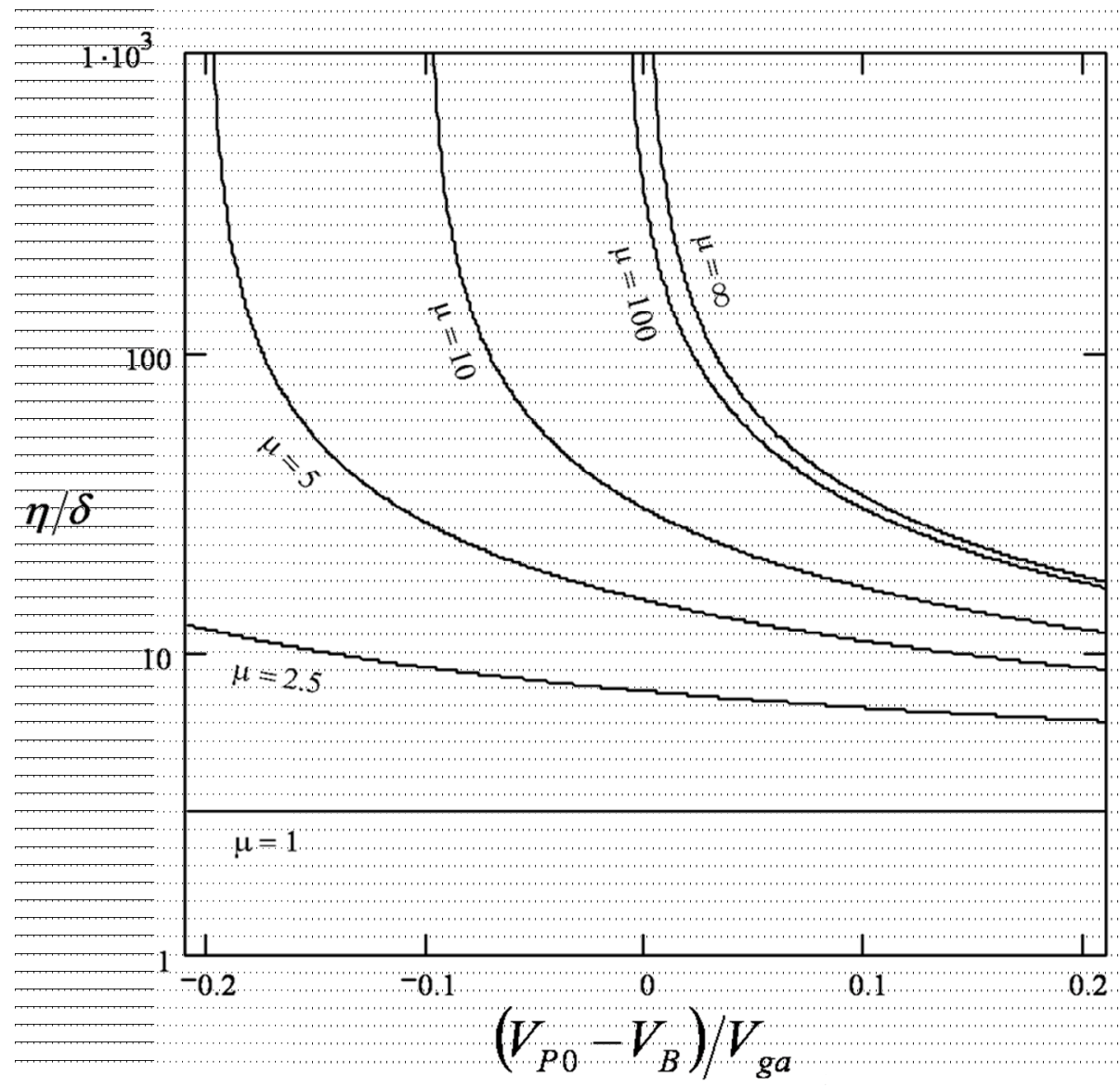

Fig. 2 


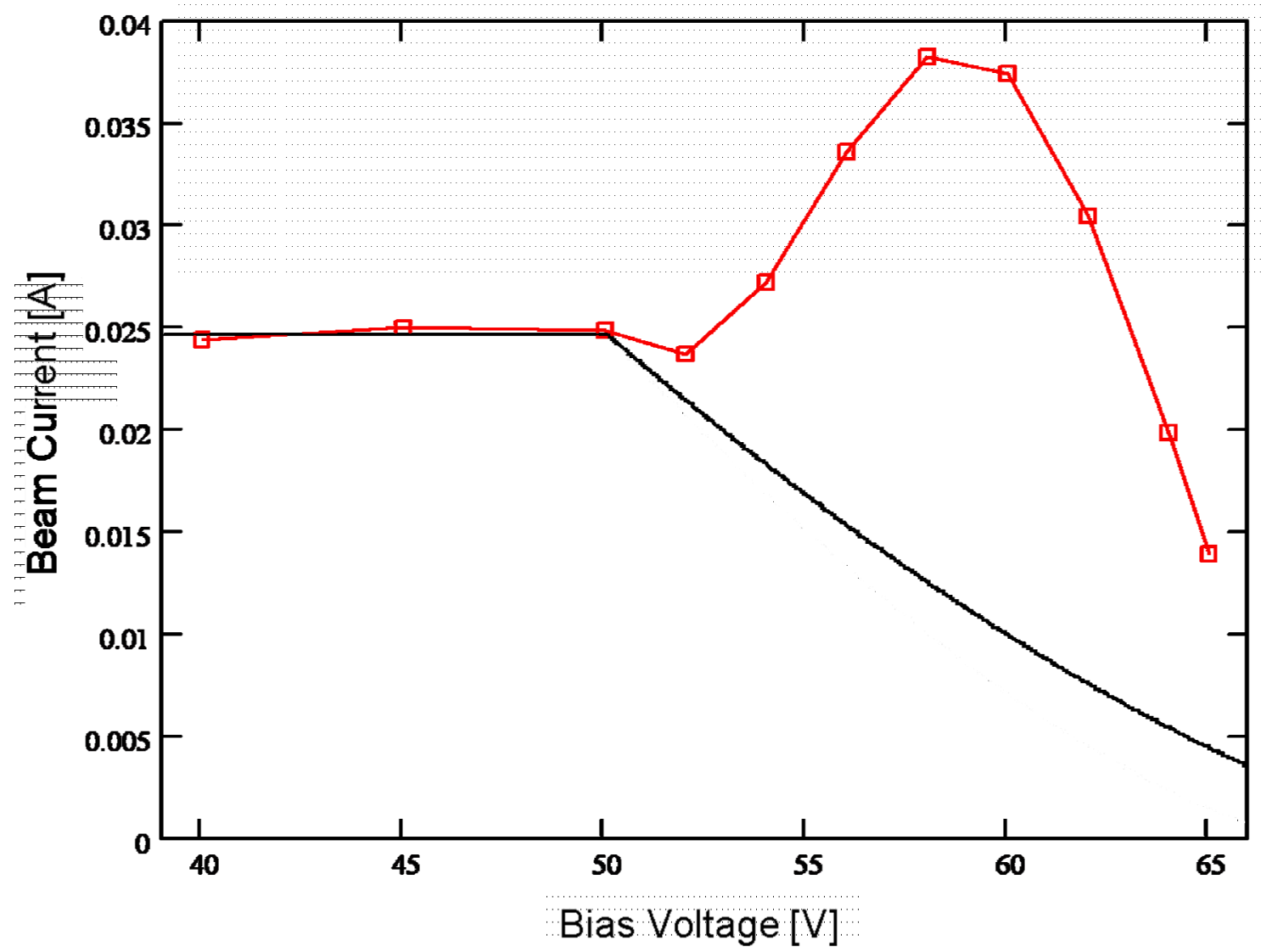

Fig. 3 

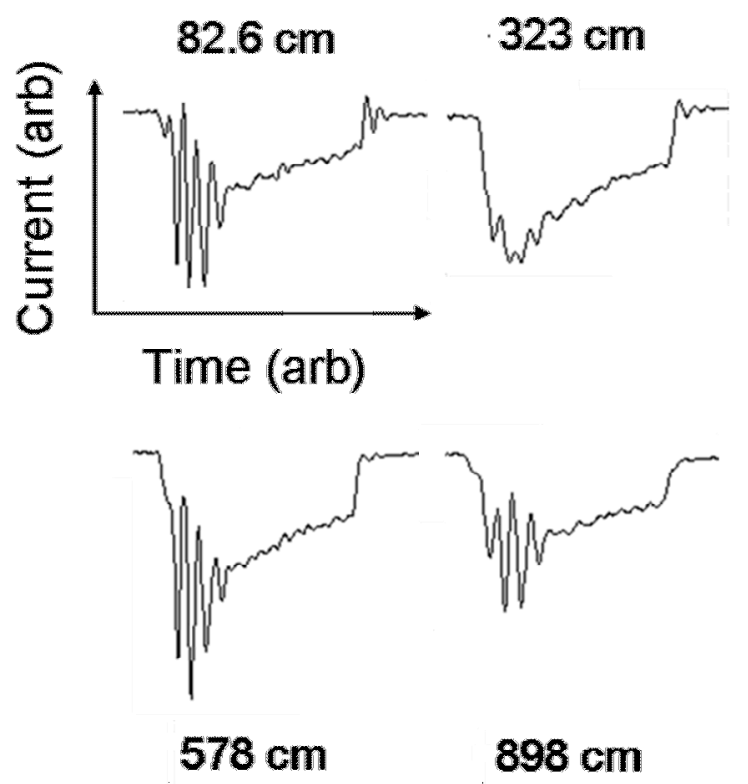

Fig. 4 


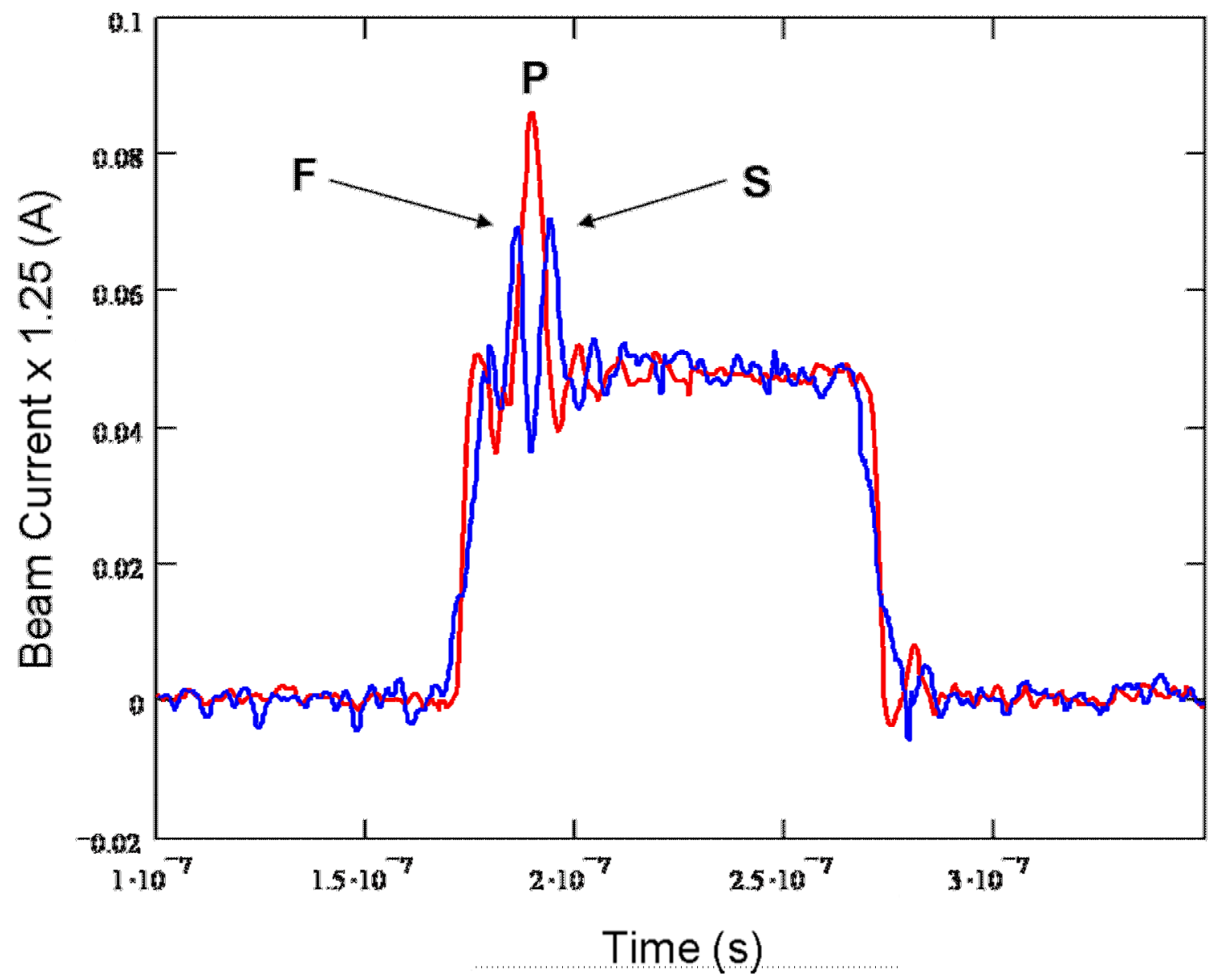

Fig. 5 